\title{
Ultrafast Charge Separation at CdS Quantum Dot/Rhodamine B molecule Interface
}

Abdelaziz Boulesbaa, Abey Issac, Dave Stockwell, Zhuangqun Huang, Jier Huang, Jianchang Guo and Tianquan Lian*

Department of Chemistry, Emory University, Atlanta, Georgia 30322, USA

\section{Ultrafast Transient Absorption Measurements:}

The visible pump-probe set up used for our studies was based on a regeneratively amplified femtosecond Ti:sapphire laser system $(1 \mathrm{kHz}$ repetition rate at $800 \mathrm{~nm}, 100 \mathrm{fs}$, $950 \mu \mathrm{J} / \mathrm{pulse}$ ). The $800 \mathrm{~nm}$ output pulse from the regenerative amplifier was split into two parts to generate pump and probe pulses. One part, with $350 \mathrm{~J} /$ pulse, was used to generate pump pulses at $400 \mathrm{~nm}$ by Second Harmonic Generation (SHG), and focused on the sample with a beam diameter of $400 \mathrm{~nm}$ and energy $130 \mathrm{~nJ} / \mathrm{pulse}$. The visible probe was generated by attenuating and focusing $\sim 10 \mu \mathrm{J}$ of the fundamental into a $2 \mathrm{~mm}$ sapphire window to produce a white light continuum with a range of $420 \mathrm{~nm}$ to $750 \mathrm{~nm}$. The probe was focused on the sample to a spot size of $100 \mu \mathrm{m}$. After the sample, the probe was focused into a fiber-coupled spectrometer (Ocean Optics USB2000, 2048 pixel $\mathrm{CCD}, \sim .25 \mathrm{~nm} / \mathrm{pixel}$ readout) and detected at a frequency of $100 \mathrm{~Hz}$. The pump pulses were synchronously blocked by a chopper at the same frequency, and the absorbance change was calculated by comparing the probe intensity with pump on and blocked. The zero time delay and instrument response function of the measurement were obtained with the instantaneous ground state bleach at $550 \mathrm{~nm}$ of Ruthenium polypyridyl complex in ethanol solution.

\section{Fluorescence lifetime measurements:}

Time-resolved fluorescence measurements were performed in the time-correlated single photon counting (TCSPC) mode under right-angle sample geometry. A femtosecond laser pulse (100 fs) with a repetition rate of $80 \mathrm{MHz}$ was generated from a mode-locked Ti:Sapphire laser (Tsunami oscillator pumped by $10 \mathrm{~W}$ Millennia Pro, Spectra-Physics). The output centered at $800 \mathrm{~nm}$ was doubled through a BBO crystal to generate $400 \mathrm{~nm}$ light that was used to excite the sample. The emission was detected by a 
Micro-channel-plate-photomultiplier tube (Hamamatsu R3809U-51), whose output was amplified and analyzed by a TCSPC board (Becker\&Hickel SPC 600).

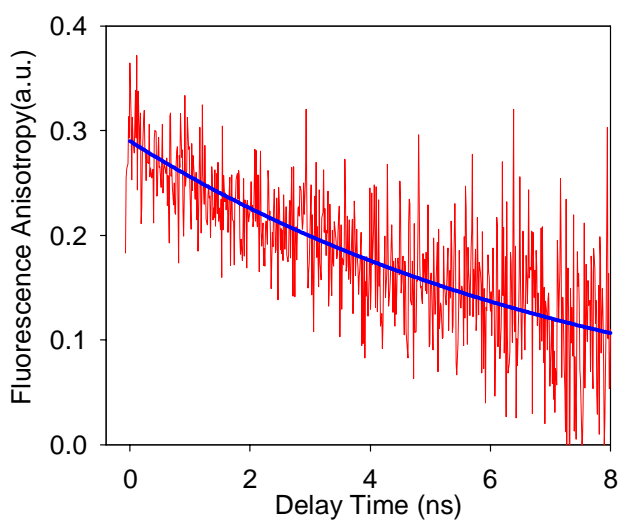

Figure S1. Fluorescence anisotropy decay of RhB/CdS in heptane. The thin redline is the experimental data and the thick blue line is a single exponential fit with amplitude of 0.29 and decay time constant of $8 \mathrm{~ns}$.

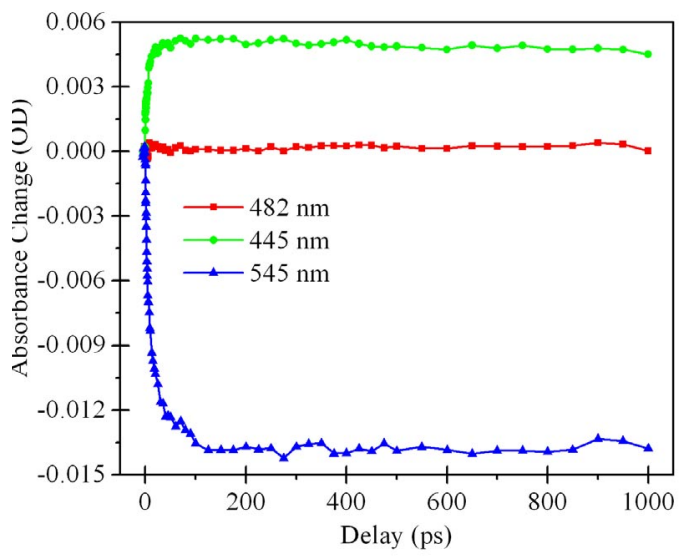

Figure S2. Transient kinetics for ground state bleach $(545 \mathrm{~nm})$, isosbestic point (482 $\mathrm{nm})$ and the anion absorption $(435 \mathrm{~nm}$ ) of RhB-CdS nanoassembly (ratio of $\mathrm{RhB} / \mathrm{CdS}=2.6$ ) recorded following $400 \mathrm{~nm}$ excitation. 


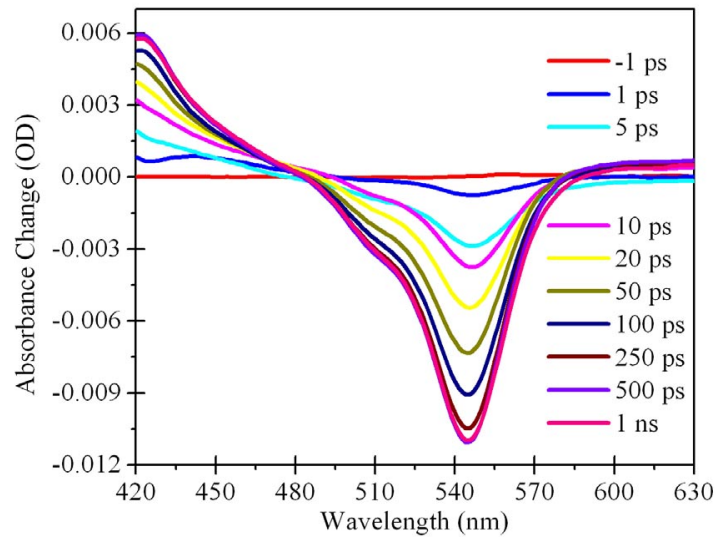

Figure S3. Transient absorption spectra of RhB-CdS nanoassembly (ratio of $\mathrm{RhB} / \mathrm{CdS}=$ 1.1) recorded at indicated delay times following $400 \mathrm{~nm}$ excitation.

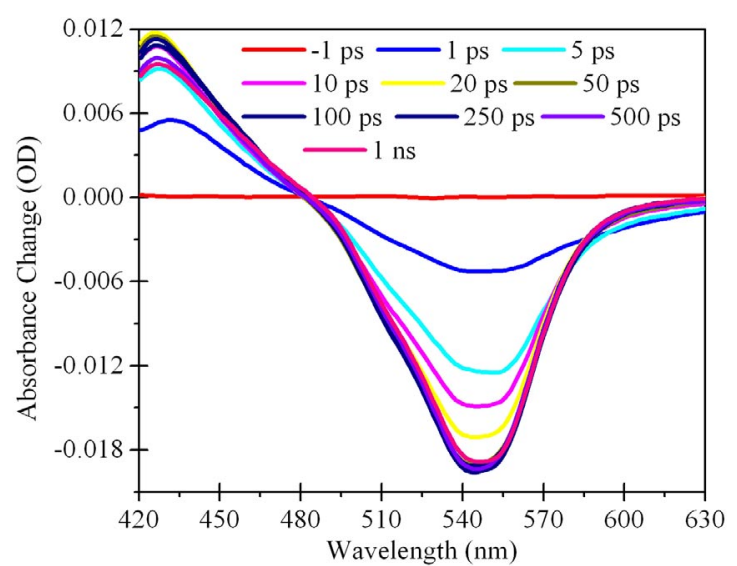

Figure S4. Transient absorption spectra of $\mathrm{RhB}-\mathrm{CdS}$ nanoassembly (ratio of $\mathrm{RhB} / \mathrm{CdS}=$ 4.1) recorded at indicated delay times following $400 \mathrm{~nm}$ excitation.

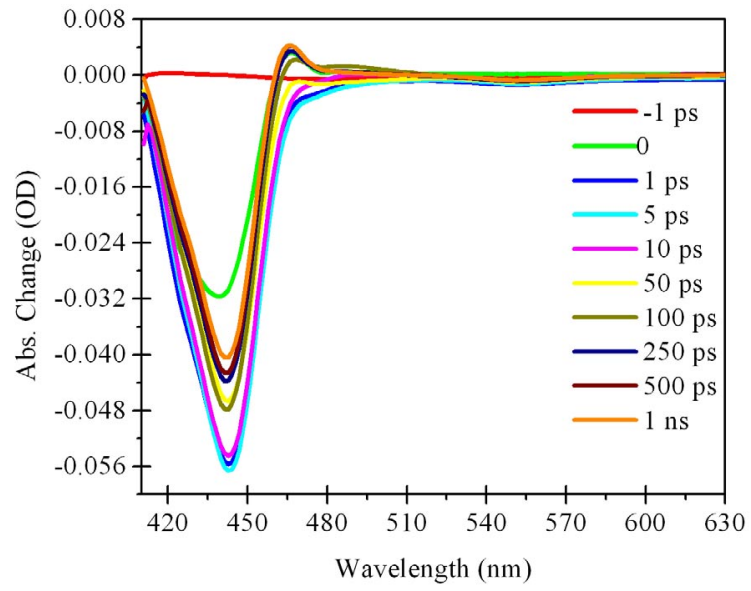


Figure S5. Transient absorption spectra of RhB-CdS (446 nm) nanoassembly (ratio of $\mathrm{RhB} / \mathrm{CdS}=4.1)$ recorded at indicated delay times following $400 \mathrm{~nm}$ excitation. $\mathrm{CdS}$ $(446 \mathrm{~nm})$ is a larger size quantum dot with the first exciton peak at $446 \mathrm{~nm}$. Unlike the smaller size CdS (387 nm), no noticeable bleach of RhB was observed. The features at around $446 \mathrm{~nm}$ are attributed to the bleach of the first exciton transition in CdS.

Table S1. Fitting parameters for kinetics traces shown in Figure 2b. The kinetics were fit to three exponential rise functions with amplitude $A_{i}$ and time constant $\tau_{i} . \tau_{\text {ave }}$ is amplitude weighted average time constant.

\begin{tabular}{|c|c|c|c|c|c|c|c|}
\hline & $\mathrm{A}_{1}$ & $\tau_{1}(\mathrm{ps})$ & $\mathrm{A}_{2}$ & $\tau_{2}(\mathrm{ps})$ & $\mathrm{A}_{3}$ & $\tau_{3}(\mathrm{ps})$ & $\tau_{\text {ave }}(\mathrm{ps})$ \\
\hline $\mathrm{R}=1.1$ & 0.2 & 3.6 & 0.47 & 50.0 & 0.33 & 200.0 & 90.2 \\
\hline $\mathrm{R}=2.6$ & 0.3 & 2.2 & 0.5 & 14.7 & 0.2 & 52.6 & 18.5 \\
\hline $\mathrm{R}=4.1$ & 0.31 & 1.1 & 0.49 & 8.3 & 0.2 & 37.0 & 11.8 \\
\hline
\end{tabular}

